\title{
Local Solutions to a Class of Parabolic System Related to the P-Laplacian
}

\author{
Qitong Ou, Huashui Zhan \\ School of Applied Mathematics, Xiamen University of Technology, Xiamen, China \\ Email: ouqitong@xmut.edu.cn
}

How to cite this paper: Ou, Q.T. and Zhan, H.S. (2016) Local Solutions to a Class of Parabolic System Related to the P-Laplacian. Advances in Pure Mathematics, 6, 868877.

http://dx.doi.org/10.4236/apm.2016.612065

Received: September 27, 2016

Accepted: November 14, 2016

Published: November 17, 2016

Copyright $\odot 2016$ by authors and Scientific Research Publishing Inc. This work is licensed under the Creative Commons Attribution International License (CC BY 4.0).

http://creativecommons.org/licenses/by/4.0/

\begin{abstract}
In this paper, the existence and uniqueness of local solutions to the initial and boundary value problem of a class of parabolic system related to the p-Laplacian are studied. The regularization method is used to construct a sequence of approximation solutions, with the help of monotone iteration technique, then we get the existence of solution of a regularized system. By the use of a standard limiting process, the existence of the local solutions of the system is obtained. Finally, the uniqueness of the solution is also proven.
\end{abstract}

\section{Keywords}

Existence, Uniqueness, Evolution, P-Laplacian, Parabolic System

\section{Introduction}

The objective of this paper is to study the existence and uniqueness of local solutions to the initial and boundary value problem of the parabolic system

$$
\begin{aligned}
& u_{i t}-\operatorname{div}\left(\left|\nabla u_{i}\right|^{p_{i}-2} \nabla u_{i}\right)=f_{i}\left(x, t, u_{1}, u_{2}, u_{3}\right),(x, t) \in \Omega_{T}, \\
& u_{i}(x, 0)=u_{i 0}(x), x \in \Omega, \\
& u_{i}(x, t)=0,(x, t) \in \partial \Omega \times(0, T),
\end{aligned}
$$

where $p_{i}>2, i=1,2,3, \quad \Omega_{T}=\Omega \times(0, T), \Omega \subset R^{n}$ is a bounded domain with smooth boundary $\partial \Omega$. The conditions of $f_{i}$ and $u_{i 0}$ will be given later.

System (1.1) is popular applied in non-Newtonian fluids [1] [2] and nonlinear filtration [3], etc. In the non-Newtonian fluids theory, $p_{i}(i=1,2,3)$ are all characteristic quantity of the medium. Media with $p_{i}>2(i=1,2,3)$ are called dilatant fluids and those with $p_{i}<2(i=1,2,3)$ are called pseudoplastics. If $p_{i}=2(i=1,2,3)$, they 
are Newtonian fluids.

Some authors have studied the global finiteness of the solutions (see [4] [5]) and blow-up properties of the solutions (see [6]) with various boundary conditions to the systems of evolutionary Laplacian equations. Zhao [7] and Wei-Gao [8] studied the existence and blow-up property of the solutions to a single equation and the systems of two equations. We found that the method of [8] can be extended to the general systems of $n$ equations. For the sake of simplicity, this paper only makes a detailed discussion on $n=3$. Since the system is coupled with nonlinear terms, it is in general difficult to study the system. In this paper, we consider some special cases by stating some methods of regularization to construct a sequence of approximation solutions with the help of monotone iteration technique and obtain the existence of solutions to a regularized system of equations. Then we obtain the existence of solutions to the system (1.1)-(1.3) by a standard limiting process. Systems (1.1) degenerates when $u_{i}=0$ or $\nabla u_{i}=0$. In general, there would be no classical solutions and hence we have to study the generalized solutions to the problem (1.1)-(1.3).

The definition of generalized solutions in this work is the following.

Definition 1.1. Function $u=\left(u_{1}, u_{2}, u_{3}\right)$ is called a generalized solution of the system (1.1)-(1.3) if $u_{i} \in L^{\infty}\left(\Omega_{T}\right) \cap L^{p_{i}}\left(0, T ; W_{0}^{1, p_{i}}(\Omega)\right), u_{i t} \in L^{2}\left(\Omega_{T}\right), i=1,2,3$, and satisfies

$$
\begin{aligned}
& \iint_{\Omega_{T}}\left(-u_{i} \varphi_{i t}+\left|\nabla u_{i}\right|^{p_{i}-2} \nabla u_{i} \nabla \varphi_{i}\right) \mathrm{d} x \mathrm{~d} t-\int_{\Omega} u_{i 0} \varphi_{i}(x, 0) \mathrm{d} x \\
& =\iint_{\Omega_{T}} f_{i}\left(x, t, u_{1}, u_{2}, u_{3}\right) \varphi_{i} \mathrm{~d} x \mathrm{~d} t,
\end{aligned}
$$

for any $\varphi_{i} \in C^{1}\left(\overline{\Omega_{T}}\right), \varphi_{i}(x, T)=0, \varphi_{i}(x, t)=0$, for $(x, t) \in \partial \Omega \times(0, T), i=1,2,3$.

Equations (4) implies that

$$
\begin{aligned}
& \int_{0}^{t} \int_{\Omega}\left(-u_{i} \varphi_{i t}+\left|\nabla u_{i}\right|^{p_{i}-2} \nabla u_{i} \nabla \varphi_{i}\right) \mathrm{d} x \mathrm{~d} t+\int_{\Omega} u_{i}(x, t) \varphi_{i}(x, t) \mathrm{d} x-\int_{\Omega} u_{i 0} \varphi_{i}(x, 0) \mathrm{d} x \\
& =\int_{0}^{t} \int_{\Omega} f_{i}\left(x, t, u_{1}, u_{2}, u_{3}\right) \varphi_{i} \mathrm{~d} x \mathrm{~d} t, \quad \text { a.e. } t \in(0, T) .
\end{aligned}
$$

The followings are the constrains to the nonlinear functions $f_{i}, i=1,2,3$, involved in this paper.

Definition 1.2. A function $f=f\left(u_{1}, u_{2}, u_{3}\right)$ is said to be quasimonotone nondecreasing (resp., nonincreasing) if for fixed $u_{j}(j \neq i), f$ is nondecreasing (resp., nonincreasing) in $u_{i},(i=1,2,3)$.

Our main existence result is following:

Theorem 1.3. If there exist nonnegative functions $f_{i}\left(x, t, u_{1}, u_{2}, u_{3}\right) \in C\left(\bar{\Omega} \times[0, T] \times R^{3}\right)$ which are quasimonotonically nondecreasing for $u_{1}, u_{2}, u_{3}(i=1,2,3)$, and a nonnegative function $g(s) \in C^{1}(R)$ such that

$$
\left|f_{i}\left(x, t, u_{1}, u_{2}, u_{3}\right)\right| \leq \min \left\{g\left(u_{1}\right), g\left(u_{2}\right), g\left(u_{3}\right)\right\} \text { and } u_{i 0} \in L^{\infty}(\Omega) \cap W_{0}^{1, p_{i}}(\Omega) .
$$

Then there exists a constant $T^{\prime} \in[0, T]$ such that the system (1.1)-(1.3) has a solution $u=\left(u_{1}, u_{2}, u_{3}\right)$ in the sence of Definition 1.1 with $T$ replaced by $T^{\prime}$.

In Theorem 1.3, we just obtain the existence of local solution. As known to all, when the system degenerates into an equation, as long as some order of growth conditions is 
added on $f$, we can find the global solution, which is the main result of [7]. The existence of the global solution of (1.1)-(1.3) remains to be further studied.

On the other hand, similar to [8], we made the assumption of monotonicity to $f_{i}$. From the current point of view, the condition is relatively strong. It is well worth studying how to reduce $f_{i}$ monotonicity requirements of the system (1.1)-(1.3).

\section{Proof of Theorem 1.3}

To prove the theorem, we consider the following regularized problem

$$
\begin{aligned}
& u_{i t}-\operatorname{div}\left(\left(\left|\nabla u_{i}\right|^{2}+\varepsilon\right)^{\frac{p_{i}-2}{2}} \nabla u_{i}\right)=f_{i \varepsilon}\left(x, t, u_{1}, u_{2}, u_{3}\right),(x, t) \in \Omega_{T}, \\
& u_{i}(x, 0)=u_{i 0 \varepsilon}(x), x \in \Omega, \\
& u_{i}(x, t)=0,(x, t) \in \partial \Omega \times(0, T),
\end{aligned}
$$

where $f_{i \varepsilon} \in C^{1}\left(\bar{\Omega} \times[0, T] \times R^{3}\right), \quad f_{i \varepsilon}$ are quasimonotone nondecreasing and $f_{i \varepsilon} \rightarrow f_{i}$ uniformly on bounded subsets of $\bar{\Omega} \times[0, T] \times R^{3}$, also

$$
\left|f_{i \varepsilon}\left(x, t, u_{1}, u_{2}, u_{3}\right)\right| \leq \min \left\{g\left(u_{1}\right), g\left(u_{2}\right), g\left(u_{3}\right)\right\},
$$

$u_{i 0 \varepsilon} \in C_{0}^{1}(\Omega),\left|u_{i 0 \varepsilon}\right|_{L^{p_{i}}} \leq\left|u_{i 0}\right|_{L^{p_{i}}},\left|\nabla u_{i 0 \varepsilon}\right|_{L^{p_{i}}} \leq C\left|\nabla u_{i 0}\right|_{L^{p_{i}}}, u_{i 0 \varepsilon} \rightarrow u_{i 0}$ strongly in $W_{0}^{1, p_{i}}(\Omega)$.

Lemma 2.1. The regularized problem (2.1)-(2.3) has a generalized solution.

Proof. Starting from a suitable initial iteration $\left(u_{1 \varepsilon}^{(0)}, u_{2 \varepsilon}^{(0)}, u_{3 \varepsilon}^{(0)}\right)$, we construct a sequence $\left\{\left(u_{1 \varepsilon}^{(k)}, u_{2 \varepsilon}^{(k)}, u_{3 \varepsilon}^{(k)}\right)\right\}$ from the iteration process

$$
\begin{aligned}
& u_{i \varepsilon t}^{(k)}-\operatorname{div}\left(\left(\left|\nabla u_{i \varepsilon}^{(k)}\right|^{2}+\varepsilon\right)^{\frac{p_{i}-2}{2}} \nabla u_{i \varepsilon}^{(k)}\right)=f_{i \varepsilon}\left(x, t, u_{1 \varepsilon}^{(k-1)}, u_{2 \varepsilon}^{(k-1)}, u_{3 \varepsilon}^{(k-1)}\right),(x, t) \in \Omega_{T}, \\
& u_{i \varepsilon}^{(k)}(x, 0)=u_{i 0 \varepsilon}(x), x \in \Omega, \\
& u_{i \varepsilon}^{(k)}(x, t)=0,(x, t) \in \partial \Omega \times(0, T),
\end{aligned}
$$

where $i=1,2,3$. It is clear that for each $k=1,2, \cdots$, the above system consists of three nondegenerated and uncoupled initial boundary-value problems.

By classical results (see [9]) for fixed $\varepsilon$ and $k$ the problem (2.5)-(2.7) has a classical solution $\left\{\left(u_{1 \varepsilon}^{(k)}, u_{2 \varepsilon}^{(k)}, u_{3 \varepsilon}^{(k)}\right)\right\}$ if $\left\{\left(u_{1 \varepsilon}^{(k-1)}, u_{2 \varepsilon}^{(k-1)}, u_{3 \varepsilon}^{(k-1)}\right)\right\}$ is smooth.

To ensure that this sequence converges to a solution of (2.1)-(2.3), it is necessary to choose a suitable initial iteration. The choice of this function depends on the type of quasimonotone property of $\left(f_{1}, f_{2}, f_{3}\right)$. In the following, we establish the monotone property of the sequence.

Set $\bar{u}_{i \varepsilon}^{(0)}(x, t)=\sup _{\Omega_{T}}\left\{u_{i 0 \varepsilon}(x)\right\}, i=1,2,3$. Let $\bar{u}_{i \varepsilon}^{(1)}$ be a classical solution of the following problem.

$$
\begin{aligned}
& \bar{u}_{i \varepsilon t}^{(1)}-\operatorname{div}\left(\left(\left|\nabla \bar{u}_{i \varepsilon}^{(1)}\right|^{2}+\varepsilon\right)^{\frac{p_{i}-2}{2}} \nabla \bar{u}_{i \varepsilon}^{(1)}\right)=f_{i \varepsilon}\left(x, t, \bar{u}_{1 \varepsilon}^{(0)}, \bar{u}_{2 \varepsilon}^{(0)}, \bar{u}_{3 \varepsilon}^{(0)}\right),(x, t) \in \Omega_{T}, \\
& \bar{u}_{i \varepsilon}^{(1)}(x, 0)=u_{i 0 \varepsilon} \leq \bar{u}_{i \varepsilon}^{(0)}, x \in \Omega,
\end{aligned}
$$




$$
\bar{u}_{i \varepsilon}^{(1)}(x, t)=0,(x, t) \in \partial \Omega \times(0, T),
$$

By $f_{i \varepsilon}\left(x, t, u_{1 \varepsilon}^{(0)}, u_{2 \varepsilon}^{(0)}, u_{3 \varepsilon}^{(0)}\right) \leq f_{i \varepsilon}\left(x, t, \bar{u}_{1 \varepsilon}^{(0)}, \bar{u}_{2 \varepsilon}^{(0)}, \bar{u}_{3 \varepsilon}^{(0)}\right)$ and the comparison theorem (see [10]), we have that

$$
\bar{u}_{1 \varepsilon}^{(1)} \leq \bar{u}_{1 \varepsilon}^{(0)}, \bar{u}_{2 \varepsilon}^{(1)} \leq \bar{u}_{2 \varepsilon}^{(0)} \text { and } \bar{u}_{3 \varepsilon}^{(1)} \leq \bar{u}_{3 \varepsilon}^{(0)} .
$$

Hence by the quasimonotone nondecreasing property of $f_{i \varepsilon}$, we have

$$
\begin{aligned}
& f_{i \varepsilon}\left(x, t, \bar{u}_{1 \varepsilon}^{(1)}, \bar{u}_{2 \varepsilon}^{(1)}, \bar{u}_{3 \varepsilon}^{(1)}\right) \leq f_{i \varepsilon}\left(x, t, \bar{u}_{1 \varepsilon}^{(0)}, \bar{u}_{2 \varepsilon}^{(1)}, \bar{u}_{3 \varepsilon}^{(1)}\right) \\
& \leq f_{i \varepsilon}\left(x, t, \bar{u}_{1 \varepsilon}^{(0)}, \bar{u}_{2 \varepsilon}^{(0)}, \bar{u}_{3 \varepsilon}^{(1)}\right) \leq f_{i \varepsilon}\left(x, t, \bar{u}_{1 \varepsilon}^{(0)}, \bar{u}_{2 \varepsilon}^{(0)}, \bar{u}_{3 \varepsilon}^{(0)}\right)
\end{aligned}
$$

for $i=1,2,3$.

Using the same argument as above, we can obtain a classical solution $\left(\bar{u}_{1 \varepsilon}^{(2)}, \bar{u}_{2 \varepsilon}^{(2)}, \bar{u}_{3 \varepsilon}^{(2)}\right)$ of the problem

$$
\begin{aligned}
& \bar{u}_{i \varepsilon t}^{(2)}-\operatorname{div}\left(\left(\left|\nabla \bar{u}_{i \varepsilon}^{(2)}\right|^{2}+\varepsilon\right)^{\frac{p_{i}-2}{2}} \nabla \bar{u}_{i \varepsilon}^{(2)}\right)=f_{i \varepsilon}\left(x, t, \bar{u}_{1 \varepsilon}^{(1)}, \bar{u}_{2 \varepsilon}^{(1)}, \bar{u}_{3 \varepsilon}^{(1)}\right),(x, t) \in \Omega_{T}, \\
& \bar{u}_{i \varepsilon}^{(2)}(x, 0)=u_{i 0 \varepsilon}, x \in \Omega, \\
& \bar{u}_{i \varepsilon}^{(2)}(x, t)=0,(x, t) \in \partial \Omega \times(0, T),
\end{aligned}
$$

for $i=1,2,3$.

By the comparison theorem, we have

$$
\bar{u}_{1 \varepsilon}^{(2)} \leq \bar{u}_{1 \varepsilon}^{(1)}, \bar{u}_{2 \varepsilon}^{(2)} \leq \bar{u}_{2 \varepsilon}^{(1)} \text { and } \bar{u}_{3 \varepsilon}^{(2)} \leq \bar{u}_{3 \varepsilon}^{(1)} \text {. }
$$

By induction method, we obtain a nonincreasing sequence of smooth functions

$$
\bar{u}_{i \varepsilon}^{(0)} \geq \bar{u}_{i \varepsilon}^{(1)} \geq \bar{u}_{i \varepsilon}^{(2)} \geq \cdots \geq \bar{u}_{i \varepsilon}^{(k)} \geq \cdots
$$

In a similar way, by setting $\underline{u}_{i \varepsilon}^{(0)}(x, t)=\inf _{\Omega_{T}}\left\{u_{i 0 \varepsilon}(x)\right\}, i=1,2,3$, we can get a solution $\left(\underline{u}_{1 \varepsilon}^{(1)}, \underline{u}_{2 \varepsilon}^{(1)}, \underline{u}_{3 \varepsilon}^{(1)}\right)$ of

$$
\begin{aligned}
& \underline{u}_{i \varepsilon t}^{(1)}-\operatorname{div}\left(\left(\left|\nabla \underline{u}_{i \varepsilon}^{(1)}\right|^{2}+\varepsilon\right)^{\frac{p_{i}-2}{2}} \nabla \underline{u}_{i \varepsilon}^{(1)}\right)=f_{i \varepsilon}\left(x, t, \underline{u}_{1 \varepsilon}^{(0)}, \underline{u}_{2 \varepsilon}^{(0)}, \underline{u}_{3 \varepsilon}^{(0)}\right),(x, t) \in \Omega_{T}, \\
& \underline{u}_{i \varepsilon}^{(1)}(x, 0)=u_{i 0 \varepsilon} \geq \underline{u}_{i \varepsilon}^{(0)}, x \in \Omega, \\
& \underline{u}_{i \varepsilon}^{(1)}(x, t)=0,(x, t) \in \partial \Omega \times(0, T),
\end{aligned}
$$

with

$$
\underline{u}_{1 \varepsilon}^{(1)} \geq \underline{u}_{1 \varepsilon}^{(0)}, \underline{u}_{2 \varepsilon}^{(1)} \geq \underline{u}_{2 \varepsilon}^{(0)} \text { and } \underline{u}_{3 \varepsilon}^{(1)} \geq \underline{u}_{3 \varepsilon}^{(0)}
$$

In the same way as above, we obtain a nondecreasing sequence of smooth functions

$$
\underline{u}_{i \varepsilon}^{(0)} \leq \underline{u}_{i \varepsilon}^{(1)} \leq \underline{u}_{i \varepsilon}^{(2)} \leq \cdots \leq \underline{u}_{i \varepsilon}^{(k)} \leq \cdots
$$

It is obvious that $\underline{\underline{u}}_{i \varepsilon}^{(0)} \leq \bar{u}_{i \varepsilon}^{(0)}$. By induction method, we may assume that $\underline{u}_{i \varepsilon}^{(k)} \leq \bar{u}_{i \varepsilon}^{(k)}$. Since $f_{i \varepsilon}$ is quasimonotone nondecreasing, we have 


$$
\begin{aligned}
& f_{i \varepsilon}\left(x, t, \underline{u}_{1 \varepsilon}^{(k)}, \underline{u}_{2 \varepsilon}^{(k)}, \underline{u}_{3 \varepsilon}^{(k)}\right) \leq f_{i \varepsilon}\left(x, t, \bar{u}_{1 \varepsilon}^{(k)}, \underline{u}_{2 \varepsilon}^{(k)}, \underline{u}_{3 \varepsilon}^{(k)}\right) \\
& \leq f_{i \varepsilon}\left(x, t, \bar{u}_{1 \varepsilon}^{(k)}, \bar{u}_{2 \varepsilon}^{(k)}, \underline{u}_{3 \varepsilon}^{(k)}\right) \leq f_{i \varepsilon}\left(x, t, \bar{u}_{1 \varepsilon}^{(k)}, \bar{u}_{2 \varepsilon}^{(k)}, \bar{u}_{3 \varepsilon}^{(k)}\right)
\end{aligned}
$$

for $i=1,2,3$.

$$
\begin{aligned}
& \underline{u}_{i \varepsilon t}^{(k+1)}-\operatorname{div}\left(\left(\left|\nabla \underline{u}_{i \varepsilon}^{(k+1)}\right|^{2}+\varepsilon\right)^{\frac{p_{i}-2}{2}} \nabla \underline{u}_{i \varepsilon}^{(k+1)}\right)=f_{i \varepsilon}\left(x, t, \underline{u}_{1 \varepsilon}^{(k)}, \underline{u}_{2 \varepsilon}^{(k)}, \underline{u}_{3 \varepsilon}^{(k)}\right),(x, t) \in \Omega_{T}, \\
& \bar{u}_{i \varepsilon t}^{(k+1)}-\operatorname{div}\left(\left(\left|\nabla \bar{u}_{i \varepsilon}^{(k+1)}\right|^{2}+\varepsilon\right)^{\frac{p_{i}-2}{2}} \nabla \bar{u}_{i \varepsilon}^{(k+1)}\right)=f_{i \varepsilon}\left(x, t, \bar{u}_{1 \varepsilon}^{(k)}, \bar{u}_{2 \varepsilon}^{(k)}, \bar{u}_{3 \varepsilon}^{(k)}\right),(x, t) \in \Omega_{T}, \\
& \underline{u}_{i \varepsilon}^{(k+1)}(x, 0)=u_{i 0 \varepsilon}=\bar{u}_{i \varepsilon}^{(k+1)}(x, 0), x \in \Omega, \\
& \underline{u}_{i \varepsilon}^{(k+1)}(x, t)=0=\bar{u}_{i \varepsilon}^{(k+1)}(x, t),(x, t) \in \partial \Omega \times(0, T),
\end{aligned}
$$

By the comparison principle, we have $\underline{u}_{i \varepsilon}^{(k+1)} \leq \bar{u}_{i \varepsilon}^{(k+1)}$. Therefore

$$
\underline{u}_{i \varepsilon}^{(0)} \leq \underline{u}_{i \varepsilon}^{(1)} \leq \cdots \leq \underline{u}_{i \varepsilon}^{(k)} \leq \underline{u}_{i \varepsilon}^{(k+1)} \leq \bar{u}_{i \varepsilon}^{(k+1)} \leq \bar{u}_{i \varepsilon}^{(k)} \leq \cdots \leq \bar{u}_{i \varepsilon}^{(1)} \leq \bar{u}_{i \varepsilon}^{(0)} .
$$

Taking $u_{i \varepsilon}^{(k)}=\underline{u}_{i \varepsilon}^{(k)}(i=1,2,3)$, we get a nondecreasing bounded sequence $\left\{u_{i \varepsilon}^{(k)}\right\}_{k=1}^{\infty}(i=1,2,3)$. Hence there exist functions $u_{i \varepsilon}(i=1,2,3)$ such that

$$
\lim _{k \rightarrow \infty} u_{i \varepsilon}^{(k)}=u_{i \varepsilon} \text {, a.e. in } \Omega_{T} \text {. }
$$

By the continuity of $f_{i \varepsilon}(i=1,2,3)$, we have

$$
\lim _{k \rightarrow \infty} f_{i \varepsilon}\left(x, t, u_{1 \varepsilon}^{(k)}, u_{2 \varepsilon}^{(k)}, u_{3 \varepsilon}^{(k)}\right)=f_{i \varepsilon}\left(x, t, u_{1 \varepsilon}, u_{2 \varepsilon}, u_{3 \varepsilon}\right) \text { a.e. in } \Omega_{T} .
$$

We now prove that there exist $T^{\prime} \in(0, T]$ and a constant $M$ (independent of $k$ and $\varepsilon$ ) such that for all $k$, we have

$$
\left|u_{i \varepsilon}^{(k)}\right|_{L^{\infty}\left(\Omega_{T^{\prime}}\right)} \leq M, i=1,2,3 .
$$

Let $v_{i}^{ \pm}(t)(i=1,2,3)$ be the solutions of the ordinary differential equations

$$
\frac{\mathrm{d} v_{i}^{ \pm}}{\mathrm{d} t}= \pm g\left(v_{i}\right), v_{i}^{ \pm}(0)= \pm\left|u_{i 0}\right|_{L^{\infty}(\Omega)},(i=1,2,3) .
$$

By standard results in [11], there exist $T_{i} \in(0, T), i=1,2,3$, such that $v_{i}^{ \pm}$exists on $\left[0, T_{i}\right]$ with $T_{i}$ depends only on $\left|u_{i 0}\right|_{L^{\infty}(\Omega)}$. By the comparison theorem

$$
\left|u_{i \varepsilon}^{(k)}(x, t)\right| \leq \max \left\{v_{i}^{+}(t),-v_{i}^{-}(t)\right\},(i=1,2,3) .
$$

Setting $T^{\prime}=\frac{1}{3} \min \left\{T_{1}, T_{2}, T_{3}\right\}, M=\max \left\{v_{i}^{+}\left(T^{\prime}\right),-v_{i}^{-}\left(T^{\prime}\right)\right\}$, we obtain (2.31).

We now claim that $u_{i \varepsilon}^{(k)}-u_{i \varepsilon}$, as $k \rightarrow \infty$, in $L^{p_{i}}\left(0, T^{\prime} ; W_{0}^{1, p_{i}}(\Omega)\right)(i=1,2,3)$, where $\longrightarrow$ stands for weak convergence, $i=1,2,3$.

Multiplying (2.5) by $u_{i \varepsilon}^{(k)}$ and integrating over $\Omega_{T^{\prime}}$, we obtain that 


$$
\begin{aligned}
& \iint_{\Omega_{T^{\prime}}}\left[u_{i \varepsilon t}^{(k)} u_{i \varepsilon}^{(k)}-\operatorname{div}\left(\left(\left|\nabla u_{i \varepsilon}^{(k)}\right|^{2}+\varepsilon\right)^{\frac{p_{i}-2}{2}} \nabla u_{i \varepsilon}^{(k)}\right) u_{i \varepsilon}^{(k)}\right] \mathrm{d} x \mathrm{~d} t \\
& =\iint_{\Omega_{T^{\prime}}} f_{i \varepsilon}\left(x, t, u_{1 \varepsilon}^{(k-1)}, u_{2 \varepsilon}^{(k-1)}, u_{3 \varepsilon}^{(k-1)}\right) u_{i \varepsilon}^{(k)} \mathrm{d} x \mathrm{~d} t .
\end{aligned}
$$

Furthermore

$$
\begin{aligned}
& \int_{\Omega} \int_{0}^{T^{\prime}} \frac{1}{2} \frac{\mathrm{d}}{\mathrm{d} t}\left(u_{i \varepsilon t}^{(k)}\right)^{2} \mathrm{~d} t \mathrm{~d} x+\iint_{\Omega_{T^{\prime}}}\left(\left|\nabla u_{i \varepsilon}^{(k)}\right|^{2}+\varepsilon\right)^{\frac{p_{i}-2}{2}}\left|\nabla u_{i \varepsilon}^{(k)}\right|^{2} \mathrm{~d} x \mathrm{~d} t \\
& =\iint_{\Omega_{T^{\prime}}} f_{i \varepsilon}\left(x, t, u_{1 \varepsilon}^{(k-1)}, u_{2 \varepsilon}^{(k-1)}, u_{3 \varepsilon}^{(k-1)}\right) u_{i \varepsilon}^{(k)} \mathrm{d} x \mathrm{~d} t, \text { i.e. } \\
& \iint_{\Omega_{T^{\prime}}}\left(\left|\nabla u_{i \varepsilon}^{(k)}\right|^{2}+\varepsilon\right)^{\frac{p_{i}-2}{2}}\left|\nabla u_{i \varepsilon}^{(k)}\right|^{2} \mathrm{~d} x \mathrm{~d} t \\
& =\iint_{\Omega_{T^{\prime}}} f_{i \varepsilon}\left(x, t, u_{1 \varepsilon}^{(k-1)}, u_{2 \varepsilon}^{(k-1)}, u_{3 \varepsilon}^{(k-1)}\right) u_{i \varepsilon}^{(k)} \mathrm{d} x \mathrm{~d} t \\
& -\frac{1}{2} \int_{\Omega}\left[\left(u_{i \varepsilon}^{(k)}\left(x, T^{\prime}\right)\right)^{2}-\left(u_{i \varepsilon}^{(k)}(x, 0)\right)^{2}\right] \mathrm{d} x .
\end{aligned}
$$

By (2.12) and the property of $f_{i \varepsilon}$

$$
\left|\nabla u_{i \varepsilon}^{(k)}\right|_{L^{p_{i}}\left(\Omega_{T^{\prime}}\right)}^{p_{i}} \leq \iint_{\Omega_{T^{\prime}}}\left(\left|\nabla u_{i \varepsilon}^{(k)}\right|^{2}+\varepsilon\right)^{\frac{p_{i}-2}{2}}\left|\nabla u_{i \varepsilon}^{(k)}\right|^{2} \mathrm{~d} x \mathrm{~d} t \leq C,
$$

where $C$ is a constant independent of $\varepsilon$ and $k$.

Multiplying (2.5) by $u_{i s t}^{(k)}$ and integrating over $\Omega_{T^{\prime}}$, we have

$$
\begin{aligned}
& \iint_{\Omega_{T^{\prime}}}\left[\left(u_{i \varepsilon t}^{(k)}\right)^{2}-\operatorname{div}\left(\left(\left|\nabla u_{i \varepsilon}^{(k)}\right|^{2}+\varepsilon\right)^{\frac{p_{i}-2}{2}} \nabla u_{i \varepsilon}^{(k)}\right) u_{i \varepsilon t}^{(k)}\right] \mathrm{d} x \mathrm{~d} t \\
& =\iint_{\Omega_{T^{\prime}}} f_{i \varepsilon}\left(x, t, u_{1 \varepsilon}^{(k-1)}, u_{2 \varepsilon}^{(k-1)}, u_{3 \varepsilon}^{(k-1)}\right) u_{i \varepsilon t}^{(k)} \mathrm{d} x \mathrm{~d} t .
\end{aligned}
$$

By Cauchy inequality and integrating by parts, we obtain

$$
\begin{aligned}
\iint_{\Omega_{T^{\prime}}}\left(u_{i \varepsilon t}^{(k)}\right)^{2} \mathrm{~d} x \mathrm{~d} t= & -\iint_{\Omega_{T^{\prime}}}\left(\left(\left|\nabla u_{i \varepsilon}^{(k)}\right|^{2}+\varepsilon\right)^{\frac{p_{i}-2}{2}} \nabla u_{i \varepsilon}^{(k)}\right) \nabla u_{i s t}^{(k)} \mathrm{d} x \mathrm{~d} t \\
& +\iint_{\Omega_{T^{\prime}}} f_{i \varepsilon}\left(x, t, u_{1 \varepsilon}^{(k-1)}, u_{2 \varepsilon}^{(k-1)}, u_{3 \varepsilon}^{(k-1)}\right) u_{i \varepsilon t}^{(k)} \mathrm{d} x \mathrm{~d} t \\
\leq & -\int_{\Omega} \int_{0}^{T^{\prime}} \frac{\mathrm{d}}{\mathrm{d} t}\left(\left|\nabla u_{i \varepsilon}^{(k)}\right|^{p_{i}}\right) \mathrm{d} t \mathrm{~d} x+\frac{1}{2} \iint_{\Omega_{T^{\prime}}}\left(u_{i \varepsilon t}^{(k)}\right)^{2} \mathrm{~d} x \mathrm{~d} t \\
& +\frac{1}{2} \iint_{\Omega_{T^{\prime}}} f_{i \varepsilon}^{2}\left(x, t, u_{1 \varepsilon}^{(k-1)}, u_{2 \varepsilon}^{(k-1)}, u_{3 \varepsilon}^{(k-1)}\right) \mathrm{d} x \mathrm{~d} t \\
= & -\int_{\Omega}\left(\left|\nabla u_{i \varepsilon}^{(k)}\left(x, T^{\prime}\right)\right|^{p_{i}}-\left|\nabla u_{i 0 \varepsilon}^{(k)}\right|^{p_{i}}\right) \mathrm{d} x+\frac{1}{2} \iint_{\Omega_{T^{\prime}}}\left(u_{i s t}^{(k)}\right)^{2} \mathrm{~d} x \mathrm{~d} t \\
& +\frac{1}{2} \iint_{\Omega_{T^{\prime}}} f_{i \varepsilon}^{2}\left(x, t, u_{1 \varepsilon}^{(k-1)}, u_{2 \varepsilon}^{(k-1)}, u_{3 \varepsilon}^{(k-1)}\right) \mathrm{d} x \mathrm{~d} t .
\end{aligned}
$$

Hence

$\iint_{\Omega_{T^{\prime}}}\left(u_{i s t}^{(k)}\right)^{2} \mathrm{~d} x \mathrm{~d} t \leq C_{1}\left(\int_{\Omega}\left(\left|\nabla u_{i 0 \varepsilon}^{(k)}\right|^{p_{i}}\right) \mathrm{d} x+\iint_{\Omega_{T^{\prime}}} f_{i \varepsilon}^{2}\left(x, t, u_{1 \varepsilon}^{(k-1)}, u_{2 \varepsilon}^{(k-1)}, u_{3 \varepsilon}^{(k-1)}\right) \mathrm{d} x \mathrm{~d} t\right) \leq C$ 
By (2.37) and (2.40), we obtain that there exists a subsequence of $u_{i \varepsilon}^{(k)}$ converging weakly in the following sense as $j \rightarrow \infty$.

$$
\begin{aligned}
& \nabla u_{i \varepsilon}^{(k j)} \rightarrow \nabla u_{i \varepsilon}, \text { in } L^{p_{i}}\left(\Omega_{T^{\prime}}\right) \\
& \left|\nabla u_{i \varepsilon}^{(k j)}\right|^{p_{i}-2} u_{i \varepsilon x l}^{(k j)} \rightarrow w_{i \varepsilon x l}, \text { in } L^{\frac{p_{i}}{p_{i}-1}}\left(\Omega_{T^{\prime}}\right), \text { for some } w_{i \varepsilon x l} \\
& u_{i \varepsilon t}^{(k j)} \rightarrow u_{i \varepsilon t}, \text { in } L^{2}\left(\Omega_{T^{\prime}}\right)
\end{aligned}
$$

where $\rightarrow$ stands for weak convergence, $i=1,2,3$.

From (2.29), (2.30), (2.37), (2.40) and the uniqueness of the weak limits, we have that, as $k \rightarrow \infty$,

$$
\begin{aligned}
& \nabla u_{i \varepsilon}^{(k)} \rightarrow \nabla u_{i \varepsilon}, \quad \text { in } L^{p_{i}}\left(\Omega_{T^{\prime}}\right) \\
& u_{i \varepsilon}^{(k)} \rightarrow u_{i \varepsilon}, f_{i \varepsilon}\left(x, t, u_{1 \varepsilon}^{(k)}, u_{2 \varepsilon}^{(k)}, u_{3 \varepsilon}^{(k)}\right) \rightarrow f_{i \varepsilon}\left(x, t, u_{1 \varepsilon}, u_{2 \varepsilon}, u_{3 \varepsilon}\right) \quad \text { a.e. in } \Omega_{T^{\prime}} \\
& u_{i \varepsilon t}^{(k)} \rightarrow u_{i \varepsilon t}, \quad \text { in } L^{2}\left(\Omega_{T^{\prime}}\right)
\end{aligned}
$$

We now claim that $w_{i \varepsilon x l}=\left|\nabla u_{i \varepsilon}\right|^{p_{i}-2} u_{i \varepsilon x l}, i=1,2,3$.

Multiplying (2.5) by $\left(u_{i \varepsilon}^{(k j)}-u_{i \varepsilon}\right) \phi_{i}$ and integrating over $\Omega_{T^{\prime}}$ with $\phi \in C_{0}^{1}\left(\Omega_{T^{\prime}}\right), \phi_{i} \geq 0$, we get

$$
\begin{aligned}
& \iint_{\Omega_{T^{\prime}}}\left(u_{i \varepsilon}^{(k j)}-u_{i \varepsilon}\right) \phi_{i} u_{i \varepsilon t}^{(k j)} \mathrm{d} x \mathrm{~d} t+\iint_{\Omega_{T^{\prime}}}\left(\nabla u_{i \varepsilon}^{(k j)}-\nabla u_{i \varepsilon}\right) \phi_{i}\left(\left|\nabla u_{i \varepsilon}^{(k j)}\right|^{2}+\varepsilon\right)^{\frac{p_{i}-2}{2}} \nabla u_{i \varepsilon}^{(k j)} \mathrm{d} x \mathrm{~d} t \\
& +\iint_{\Omega_{T^{\prime}}}\left(u_{i \varepsilon}^{(k j)}-u_{i \varepsilon}\right) \nabla \phi_{i}\left(\left|\nabla u_{i \varepsilon}^{(k j)}\right|^{2}+\varepsilon\right)^{\frac{p_{i}-2}{2}} \nabla u_{i \varepsilon}^{(k j)} \mathrm{d} x \mathrm{~d} t \\
& =\iint_{\Omega_{T^{\prime}}}\left(u_{i \varepsilon}^{(k j)}-u_{i \varepsilon}\right) \phi_{i} f_{i \varepsilon}\left(x, t, u_{1 \varepsilon}^{(k-1)}, u_{2 \varepsilon}^{(k-1)}, u_{3 \varepsilon}^{(k-1)}\right) \mathrm{d} x \mathrm{~d} t
\end{aligned}
$$

Hence

$$
\begin{aligned}
& \iint_{\Omega_{T^{\prime}}}\left(\nabla u_{i \varepsilon}^{(k j)}-\nabla u_{i \varepsilon}\right) \phi_{i}\left(\left|\nabla u_{i \varepsilon}^{(k j)}\right|^{2}+\varepsilon\right)^{\frac{p_{i}-2}{2}} \nabla u_{i \varepsilon}^{(k j)} \mathrm{d} x \mathrm{~d} t \\
& =\iint_{\Omega_{T^{\prime}}}\left(u_{i \varepsilon}^{(k j)}-u_{i \varepsilon}\right) \phi_{i} f_{i \varepsilon}\left(x, t, u_{1 \varepsilon}^{(k-1)}, u_{2 \varepsilon}^{(k-1)}, u_{3 \varepsilon}^{(k-1)}\right) \mathrm{d} x \mathrm{~d} t-\iint_{\Omega_{T^{\prime}}}\left(u_{i \varepsilon}^{(k j)}-u_{i \varepsilon}\right) \phi_{i} u_{i \varepsilon t}^{(k j)} \mathrm{d} x \mathrm{~d} t \\
& -\iint_{\Omega_{T^{\prime}}}\left(u_{i \varepsilon}^{(k j)}-u_{i \varepsilon}\right) \nabla \phi_{i}\left(\left|\nabla u_{i \varepsilon}^{(k j)}\right|^{2}+\varepsilon\right)^{\frac{p_{i}-2}{2}} \nabla u_{i \varepsilon}^{(k j)} \mathrm{d} x \mathrm{~d} t .
\end{aligned}
$$

Since the three terms on the right hand side of the above equality converge to 0 as $j \rightarrow \infty$. This yields that

$$
\lim _{j \rightarrow \infty} \iint_{\Omega_{T^{\prime}}}\left(\nabla u_{i \varepsilon}^{(k j)}-\nabla u_{i \varepsilon}\right) \phi_{i}\left|\nabla u_{i \varepsilon}^{(k j)}\right|^{p_{i}-2} \nabla u_{i \varepsilon}^{(k j)} \mathrm{d} x \mathrm{~d} t=0
$$

On the other hand, since $\nabla u_{i \varepsilon} \in L^{p_{i}}\left(\Omega_{T^{\prime}}\right)$, we have that

$$
\lim _{j \rightarrow \infty} \iint_{\Omega_{T^{\prime}}}\left(\nabla u_{i \varepsilon}^{(k j)}-\nabla u_{i \varepsilon}\right) \phi_{i}\left|\nabla u_{i \varepsilon}\right|^{p_{i}-2} \nabla u_{i \varepsilon} \mathrm{d} x \mathrm{~d} t=0 .
$$

Note that 


$$
\begin{aligned}
& \int_{0}^{1}\left|\nabla\left(s u_{i \varepsilon}^{(k j)}\right)+(1-s) u_{i \varepsilon}\right|^{p_{i}-2} \mathrm{~d} s\left|\nabla\left(u_{i \varepsilon}^{(k j)}-u_{i \varepsilon}\right)\right|^{2} \\
& \leq\left(\left|\nabla u_{i \varepsilon}^{(k j)}\right|^{p_{i}-2} \nabla u_{i \varepsilon}^{(k j)}-\left|\nabla u_{i \varepsilon}\right|^{p_{i}-2} \nabla u_{i \varepsilon}\right)\left(\nabla u_{i \varepsilon}^{(k j)}-\nabla u_{i \varepsilon}\right) .
\end{aligned}
$$

Following (2.50) and (2.51), we have

$$
\lim _{j \rightarrow \infty} \iint_{\Omega_{T^{\prime}}} \phi_{i}\left(\int_{0}^{1}\left|\nabla\left(s u_{i \varepsilon}^{(k j)}+(1-s) u_{i \varepsilon}\right)\right|^{p_{i}-2} \mathrm{~d} s\right)\left|\nabla\left(u_{i \varepsilon}^{(k j)}-u_{i \varepsilon}\right)\right|^{2} \mathrm{~d} x \mathrm{~d} t=0 .
$$

Since

$$
\iint_{\Omega_{T^{\prime}}} \int_{0}^{1}\left|\nabla\left(s u_{i \varepsilon}^{(k j)}+(1-s) u_{i \varepsilon}\right)\right|^{p_{i}-2} \mathrm{~d} s \mathrm{~d} x \mathrm{~d} t \leq C
$$

and

$$
\begin{aligned}
& \left.|| \nabla u_{i \varepsilon}^{(k j)}\right|^{p_{i}-2} u_{i \varepsilon x l}^{(k j)}-\left|\nabla u_{i \varepsilon}\right|^{p_{i}-2} u_{i \varepsilon x l} \mid \\
& =\left|\int_{0}^{1} \frac{\mathrm{d}}{\mathrm{d} s}\left\{\left|s \nabla u_{i \varepsilon}^{(k j)}+(1-s) \nabla u_{i \varepsilon}\right|^{p_{i}-2}\left(s u_{i \varepsilon x l}^{(k j)}+(1-s) u_{i \varepsilon x l}\right)\right\} \mathrm{d} s\right| \\
& \leq\left|\int_{0}^{1}\right| s \nabla u_{i \varepsilon}^{(k j)}+\left.(1-s) \nabla u_{i \varepsilon}\right|^{p_{i}-2}\left(u_{i \varepsilon x l}^{(k j)}-u_{i \varepsilon x l}\right) d s \mid \\
& +\left|\int_{0}^{1}\left(p_{i}-2\right)\right| s \nabla u_{i \varepsilon}^{(k j)}+\left.(1-s) \nabla u_{i \varepsilon}\right|^{p_{i}-4}\left(s u_{i \varepsilon x l}^{(k j)}+(1-s) u_{i \varepsilon x l}\right)\left(u_{i \varepsilon x l}^{(k j)}-u_{i \varepsilon x l}\right) \mathrm{d} s \mid \\
& \leq C\left|\nabla\left(u_{i \varepsilon}^{(k j)}-u_{i \varepsilon}\right)\right| \int_{0}^{1}\left|s \nabla u_{i \varepsilon}^{(k j)}+(1-s) \nabla u_{i \varepsilon}\right|^{p_{i}-2} \mathrm{~d} s,
\end{aligned}
$$

by Hölder inequality, we have

$$
\begin{aligned}
& \left|\iint_{\Omega_{T^{\prime}}}\left(\left|\nabla u_{i \varepsilon}^{(k j)}\right|^{p_{i}-2} u_{i \varepsilon x l}^{(k j)}-\left|\nabla u_{i \varepsilon}\right|^{p_{i}-2} u_{i \varepsilon x l}\right) \phi_{i} \mathrm{~d} x \mathrm{~d} t\right| \\
& \leq C\left(\iint_{\Omega_{T^{\prime}}} \phi_{i} \int_{0}^{1}\left|\nabla\left(s u_{i \varepsilon}^{(k j)}+(1-s) u_{i \varepsilon}\right)\right|^{p_{i}-2} \mathrm{~d} s\left|\nabla\left(u_{i \varepsilon}^{(k j)}-u_{i \varepsilon}\right)\right|^{2} \mathrm{~d} x \mathrm{~d} t\right)^{\frac{1}{2}} \\
& \quad \times\left(\iint_{\Omega_{T^{\prime}}} \phi_{i} \int_{0}^{1}\left|\nabla\left(s u_{i \varepsilon}^{(k j)}+(1-s) u_{i \varepsilon}\right)\right|^{p_{i}-2} \mathrm{~d} s \mathrm{~d} x \mathrm{~d} t\right)^{\frac{1}{2}} \rightarrow 0, j \rightarrow \infty .
\end{aligned}
$$

i.e.,

$$
\iint_{\Omega_{T^{\prime}}}\left(w_{i \varepsilon x l}-\left|\nabla u_{i \varepsilon}\right|^{p_{i}-2} u_{i \varepsilon x l}\right) \phi_{i} \mathrm{~d} x \mathrm{~d} t \text {, for any } \phi_{i} .
$$

Hence

$$
w_{i \varepsilon x l}=\left|\nabla u_{i \varepsilon}\right|^{p_{i}-2} u_{i \varepsilon x l}, i=1,2,3 .
$$

This proves that any weak convergence subsequence of $\left|\nabla u_{i \varepsilon}^{(k)}\right|^{p_{i}-2} u_{i \varepsilon x l}^{(k)}, i=1,2,3$ will have $w_{i \varepsilon x l}$ as its weak limit and hence by a standard argument, we have that as $k \rightarrow \infty$,

$$
\left|\nabla u_{i \varepsilon}^{(k)}\right|^{p_{i}-2} u_{i \varepsilon x l}^{(k)} \rightarrow\left|\nabla u_{i \varepsilon}\right|^{p_{i}-2} u_{i \varepsilon x l}, \quad \text { in } \frac{p_{i}}{p_{i}-1}\left(\Omega_{T^{\prime}}\right), i=1,2,3 .
$$

Combining the above results, we have proved that $u_{\varepsilon}=\left(u_{1 \varepsilon}, u_{2 \varepsilon}, u_{3 \varepsilon}\right),(x, t) \in \Omega_{T^{\prime}}$ is a generalized solution of (2.1)-(2.3). 
Proof of theorem 1.3.

Since $u_{i \varepsilon}$ satisfy similar estimates as (2.31), (2.37) and (2.40), combining the property of $f_{i \varepsilon}$, we know that there are functions $u_{i} \in L^{p_{i}}\left(0, T^{\prime} ; W_{0}^{1, p_{i}}(\Omega)\right)(i=1,2,3)$ (as $\varepsilon \rightarrow 0)$ such that for some subsequence of $\left(u_{1 \varepsilon}, u_{2 \varepsilon}, u_{3 \varepsilon}\right)$, denoted again by $u_{i \varepsilon}$,

$$
\begin{aligned}
& \nabla u_{i \varepsilon} \rightarrow \nabla u_{i}, \quad \text { in } L^{p_{i}}\left(\Omega_{T^{\prime}}\right) \\
& u_{i \varepsilon} \rightarrow u_{i}, f_{i \varepsilon}\left(x, t, u_{1 \varepsilon}, u_{2 \varepsilon}, u_{3 \varepsilon}\right) \rightarrow f_{i}\left(x, t, u_{1}, u_{2}, u_{3}\right) \text { a.e. in } \Omega_{T^{\prime}} \\
& u_{i \varepsilon t} \rightarrow u_{i t}, \quad \text { in } L^{2}\left(\Omega_{T^{\prime}}\right) \\
& \left|\nabla u_{i \varepsilon}\right|^{p_{i}-2} u_{i \varepsilon x l} \rightarrow w_{i x l}, \quad \text { in } L^{\frac{p_{i}}{p_{i}-1}}\left(\Omega_{T^{\prime}}\right)
\end{aligned}
$$

In a similar way as above, we prove that $w_{i x l}=\left|\nabla u_{i}\right|^{p_{i}-2} u_{i x l}, i=1,2,3$.

By a standard limiting process, we obtain that $u=\left(u_{1}, u_{2}, u_{3}\right)$ satisfies the initial and boundary value conditions and the integrating expression. Thus $u=\left(u_{1}, u_{2}, u_{3}\right)$ is a generalized solution of (1.1)-(1.3).

\section{Uniqueness Result to the Solution of the System}

We now prove the uniqueness result to the solution of the system.

Theorem 3.1. Assume $f=\left(f_{1}, f_{2}, f_{3}\right)$ is Lipschitz continuous in $\left(u_{1}, u_{2}, u_{3}\right)$, then the solution of $(1.1)-(1.3)$ is unique.

Proof. Assume that $u=\left(u_{1}, u_{2}, u_{3}\right)$ and $v=\left(v_{1}, v_{2}, v_{3}\right)$ are two solutions of (1.1)(1.3). Let $w_{i}=u_{i}-v_{i}, i=1,2,3$, then following (1.5),

$$
\begin{aligned}
& \int_{0}^{t} \int_{\Omega}\left(-u_{i} w_{i t}+\left|\nabla u_{i}\right|^{p_{i}-2} \nabla u_{i} \nabla w_{i}\right) \mathrm{d} x \mathrm{~d} t+\int_{\Omega} u_{i}(x, t) w_{i}(x, t) \mathrm{d} x-\int_{\Omega} u_{i 0} w_{i}(x, 0) \mathrm{d} x \\
& =\int_{0}^{t} \int_{\Omega} f_{i}\left(x, t, u_{1}, u_{2}, u_{3}\right) w_{i} \mathrm{~d} x \mathrm{~d} t, \quad \text { a.e. } \quad t \in(0, T) . \\
& \int_{0}^{t} \int_{\Omega}\left(-v_{i} w_{i t}+\left|\nabla v_{i}\right|^{p_{i}-2} \nabla v_{i} \nabla w_{i}\right) \mathrm{d} x \mathrm{~d} t+\int_{\Omega} v_{i}(x, t) w_{i}(x, t) \mathrm{d} x-\int_{\Omega} v_{i 0} w_{i}(x, 0) \mathrm{d} x \\
& =\int_{0}^{t} \int_{\Omega} f_{i}\left(x, t, v_{1}, v_{2}, v_{3}\right) w_{i} \mathrm{~d} x \mathrm{~d} t, \quad \text { a.e. } \quad t \in(0, T) .
\end{aligned}
$$

By (3.1) subtracting (3.2), we get

$$
\begin{aligned}
\frac{1}{2} \int_{\Omega}\left(u_{i}-v_{i}\right)^{2} \mathrm{~d} x= & -\int_{0}^{t} \int_{\Omega}\left(\left|\nabla u_{i}\right|^{p_{i}-2} \nabla u_{i}-\left|\nabla v_{i}\right|^{p_{i}-2} \nabla v_{i}\right) \nabla\left(u_{i}-v_{i}\right) \mathrm{d} x \mathrm{~d} t \\
& +\int_{0}^{t} \int_{\Omega}\left(f_{i}\left(x, t, u_{1}, u_{2}, u_{3}\right)-f_{i}\left(x, t, v_{1}, v_{2}, v_{3}\right)\right)\left(u_{i}-v_{i}\right) \mathrm{d} x \mathrm{~d} t . \\
\leq & \int_{0}^{t} \int_{\Omega}\left(f_{i}\left(x, t, u_{1}, u_{2}, u_{3}\right)-f_{i}\left(x, t, v_{1}, v_{2}, v_{3}\right)\right)\left(u_{i}-v_{i}\right) \mathrm{d} x \mathrm{~d} t .
\end{aligned}
$$

By the inequality (3.3) and the Lipschitz condition, a simple calculation shows that

$$
\begin{aligned}
& \int_{\Omega}\left(\left|u_{1}-v_{1}\right|^{2}+\left|u_{2}-v_{2}\right|^{2}+\left|u_{3}-v_{3}\right|^{2}\right) \mathrm{d} x \\
& \leq 2 K \int_{0}^{t} \int_{\Omega}\left(\left|u_{1}-v_{1}\right|+\left|u_{2}-v_{2}\right|+\left|u_{3}-v_{3}\right|\right)^{2} \mathrm{~d} x \mathrm{~d} t \\
& \leq 6 K \int_{0}^{t} \int_{\Omega}\left(\left|u_{1}-v_{1}\right|^{2}+\left|u_{2}-v_{2}\right|^{2}+\left|u_{3}-v_{3}\right|^{2}\right) \mathrm{d} x \mathrm{~d} t
\end{aligned}
$$

Setting $F(t)=\int_{0}^{t} \int_{\Omega}\left(\left|u_{1}-v_{1}\right|^{2}+\left|u_{2}-v_{2}\right|^{2}+\left|u_{3}-v_{3}\right|^{2}\right) \mathrm{d} x \mathrm{~d} t$, then (3.4) can be written as $F^{\prime}(t) \leq 6 K F(t)$. Since $F(0) \equiv 0$, by a standard argument, we have $F(t) \equiv 0$, and 
hence $u_{i} \equiv v_{i}(i=1,2,3)$.

\section{References}

[1] Astrita, G. and Marrucci, G. (1974) Principles of Non-Newtonian Fluid Mechanics. McGrawHill, New York.

[2] Martinson, L.K. and Pavlov, K.B. (1971) Unsteady Shear Flows of a Conducting Fluid with a Rheological Power Law. Magnitnaya Gidrodinamika, 2, 50-58.

[3] Esteban, J.R. and Vazquez, J.L. (1982) On the Equation of Turbulent Filteration in OneDimensional Porous Media. Nonlinear Analysis, 10, 1303-1325.

http://dx.doi.org/10.1016/0362-546X(86)90068-4

[4] Constantin, A., Escher, J. and Yin, Z. (2004) Global Solutions for Quasilinear Parabolic System. Journal of Differential Equations, 197, 73-84.

http://dx.doi.org/10.1016/S0022-0396(03)00165-7

[5] Dichstein, F. and Escobedo, M. (2001) A Maximum Principle for Semilinear Parabolic Systems and Application. Nonlinear Analysis, 45, 825-837. http://dx.doi.org/10.1016/S0362-546X(99)00419-8

[6] Pierre, M. and Schmidt, D. (1997) Blowup in Reaction-Diffusion Systems with Dissipation of Mass. SIAM Journal on Mathematical Analysis, 28, 259-269.

http://dx.doi.org/10.1137/S0036141095295437

[7] Zhao, J. (1993) Existence and Nonexistence of Solutions for $u_{t}-\operatorname{div}\left(|\nabla u|^{p-2} \nabla u\right)=f(\nabla u, u, x, t)$. Journal of Mathematical Analysis and Applications, 172, 130-146. http://dx.doi.org/10.1006/jmaa.1993.1012

[8] Wei, Y. and Gao, W. (2007) Existence and Uniqueness of Local Solutions to a Class of Quasilinear Degenerate Parabolic Systems. Applied Mathematics and Computation, 190, 12501257. http://dx.doi.org/10.1016/j.amc.2007.02.007

[9] Ladyzenskaja, O.A., Solonnikov, V.A. and Ural'ceva, N.N. (1968) Linear and Quasilinear Equations of Parabolic Type. American Mathematical Society, Providence, RI.

[10] Friedman, A. (1964) Partial Differential Equations of Parabilic Type. Prentice-Hall Inc., Englewood Cliffs, NJ.

[11] Coddingtin, E. and Levinson, N. (1955) Theory of Ordinary Differential Equations. McGrawHill, New York. 
Submit or recommend next manuscript to SCIRP and we will provide best service for you:

Accepting pre-submission inquiries through Email, Facebook, LinkedIn, Twitter, etc.

A wide selection of journals (inclusive of 9 subjects, more than 200 journals)

Providing 24-hour high-quality service

User-friendly online submission system

Fair and swift peer-review system

Efficient typesetting and proofreading procedure

Display of the result of downloads and visits, as well as the number of cited articles

Maximum dissemination of your research work

Submit your manuscript at: http://papersubmission.scirp.org/

Or contact apm@scirp.org 